\title{
Bosonic Fractional Quantum Hall States in Rotating Optical Lattices: Projective Symmetry Group Analysis
}

\author{
T. Đurić and A. Lazarides \\ Max Planck Institute for the Physics of Complex Systems, Nöthnitzer Str. 38, 01187 Dresden, Germany
}

(Dated: June 14, 2021)

\begin{abstract}
We study incompressible ground states of bosons in a two-dimensional rotating square optical lattice. The system can be described by the Bose-Hubbard model in an effective uniform magnetic field present due to the lattice rotation. To study ground states of the system, we map it to a frustrated spin model, followed by Schwinger boson mean field theory and projective symmetry group analysis. Using symmetry analysis we identify bosonic fractional quantum Hall states, predicted for bosonic atoms in rotating optical lattices, with possible stable gapped spin liquid states within the Schwinger boson formalism. In particular, we find that previously found fractional quantum Hall states induced by the lattice potential, and with no counterpart in the continuum [G. Möller, and N. R. Cooper, Phys. Rev. Lett. 103, 105303 (2009)], correspond to " $\pi$ flux" spin liquid states of the frustrated spin model.
\end{abstract}

PACS numbers: 67.85.-d, 73.43.-f, 75.10.Kt, 75.10.Jm

\section{INTRODUCTION}

Ultracold atoms in optical lattices have provided new opportunities to experimentally realise and study a wide range of complex quantum models under controllable conditions $\stackrel{-1-3}{-3}$ Many studies of atomic systems in artificial gauge potentials were motivated by the possibility to realize strongly correlated fractional quantum Hall (FQH) states $\stackrel{4-15}{-15}$ On the other hand, quantum Hall effects $\underline{16}-18$ have attracted much attention since their discovery, and are still a very active field of study. In addition to being intrinsically interesting systems, they are also being investigated from the point of view of topological quantum computing: excitations in some FQH systems exhibit fractional, anyonic statistics and can potentially perform fault-tolerant quantum computation $\underline{\underline{19,20}}$

An artificial gauge field for neutral atoms can be created, for example, by rotating the lattice $, 4,5,8,21-23$ The rotation of the system is equivalent, in the corotating frame, to the introduction of an effective magnetic field proportional to the rotation frequency $\stackrel{4,5}{*}$ However, the system enters the FQH regime at a very high rotation frequency or a low atomic density which are hard to achieve experimentally. This has motivated development of many alternative schemes $2,6,24-33$ to introduce a vector potential to a system of neutral atoms, for example, using Raman assisted hopping combined with lattice acceleration or an inhomogeneous static electric field, $, 2,24$ or using oscillating quadrupole fields $\underline{\underline{6}}$

Prior to studies of FQH states in optical lattices, such states have been predicted for rotating Bose-Einstein condensates in harmonic magnetic traps $\underset{7,8}{7}$ The rotation (effective magnetic field) introduces vortices into the condensate and eventually, at sufficiently high rotation frequency, leads to formation of an Abrikosov vortex lattice $\stackrel{4,34}{ }$ When the number of vortices becomes comparable to the number of bosons the system can enter into a FQH state. However, since the interactions among the bosonic atoms in the magnetic traps are weak, the FQH ground state is separated from the excited states by a small energy gap. On the other hand, in optical lattices interaction energies among the atoms are much larger due to confinement of atoms in a smaller volume. This leads to more robust FQH states separated from the excited states by much larger energy gaps than for the atoms in harmonic traps. In addition, the presence of the lattice leads to new interesting physical effects. In particular, new FQH states with no counterpart in the continuum limit 13 were predicted for systems of bosons in the presence of the lattice potential and effective magnetic field.

In the presence of a tight-binding lattice and a uniform magnetic field the single-particle energy levels change from simple Landau levels in the absence of the lattice into the Hofstadter butterfly spectrum 35,36 The energy spectrum depends on the magnetic flux $\alpha$ per lattice plaquette (measured in units of the flux quantum $\phi_{0}=2 \pi \hbar / Q$, where $Q$ is the effective charge of the particle).

For rational $\alpha=p / q$ (with $p$ and $q$ coprime) the spectrum splits into $q$ bands and each state is $q$ fold degenerate. When the single-particle energies are plotted against the flux $\alpha$ the resulting Hofstadter butterfly has a fractal structure. For $\alpha \ll 1$ the low-lying bands become the Landau levels of the continuum. For bosonic atoms with repulsive interactions, in the presence of a lattice and a uniform effective magnetic field with $\alpha \ll 1$ one thus expects to find the same states as in a continuum bosonic quantum Hall system. Some of the states that appear in the continuum are, for example, bosonic $\nu=1 / 2$ Laughlin state and $\nu=1,3 / 2, \ldots$ Read-Rezayi states $37-39$ with $\nu=n / \alpha$ and $n$ being the average particle density per lattice site. It has been shown that at sufficiently low particle density the lattice has a negligible effect on the nature of the continuum $\nu=1 / 2$ state .11 However, in the presence of the lattice additional novel FQH states with no counterpart in the continuum limit have also been predicted 13

In the present paper we study incompressible ground 
states of interacting bosons in a two dimensional rotating square optical lattice. Bosonic atoms in a deep optical lattice can be described by a Bose-Hubbard model. An effective vector potential, due to the lattice rotation, introduces an Aharonov-Bohm phase for the bosons hopping from site to site and the complex tunelling couplings appear in the Hubbard Hamiltonian. In the regime of strong on-site interaction the Bose-Hubbard Hamiltonian can be mapped to an effective frustrated spin model. To study the spin model, we use Schwinger boson mean field theory ${ }^{40}-43$ and projective symmetry group (PSG) analysis. $44-48$

Schwinger boson mean-field theory allows description of both ordered and disordered phases, that is, the resulting mean field Hamiltonian does not have any preferred direction in spin space. Magnetic ordering is identified as Bose-condensation of Schwinger bosons. For such condensed phases the energy excitation spectrum is gapless, while a gapped spectrum corresponds to incompressible spin liquid phases. In this work, we focus on the possible noncondensed ground states of the system, which do not break any symmetries. For such states, PSG anlysis can be used to determine different mean field ansätze that reflect all the physical symmetries of the system and to classify possible spin liquid states.

Unlike conventional states which can be distinguished by patterns of broken symmetry, different spin liquid phases that are completely symmetric can be distinguished by considering how the symmetries are realized in those phases. Such symmetric spin liquid phases can be classified using PSG analysis. The main idea of the PSG analysis of the mean field states in the Schwinger boson theory is that the mean field ansatz preserves all the symmetries of the spin Hamiltonian when it is invariant under transformations that are combined physical symmetry and $U(1)$ gauge group transformations. In other words, symmetries of the spin model can be preserved in the mean field state in some indirect way and invariance of the mean field ansatz under all symmetry transformations is a sufficient, however not necessary condition. The set of all transformations that leave a mean field ansatz invariant is called the PSG.

Different allowed sets of combined transformations can be found by considering algebraic relations among symmetry group elements. Those different allowed sets of combined transformations then characterize physically distinct symmetric spin states. The bosonic FQH states of the system can then be identified with different spin liquid states of the effective spin model. In particular, our results show that lattice induced FQH states of the system correspond to " $\pi$ flux" spin liquid states of the effective spin model.

The paper is organized as follows. In Sec. II we introduce the Bose-Hubbard model for the system and outline the mapping to the quantum spin model. In Sec. III we describe Schwinger boson mean field theory for the effective quantum spin model. The magnetic symmetry group operators that correspond to the symmetries of the sys- tem are defined in Sec. IV. In Sec. V we find possible noncondensed spin liquid ground states of the system using PSG analysis. Relation between the spin liquid states found in Sec. $\mathrm{V}$ and bosonic FQH states of the system is investigated in Sec. VI, In the final section, we draw our conclusions.

\section{EFFECTIVE FRUSTRATED SPIN HAMILTONIAN}

We consider the system of bosonic atoms in a twodimensional optical lattice and in the presence of an effective vector potential introduced, for example, through lattice rotation. In the limit of weak tunneling $t$ between wells within the lattice, compared to the level spacing in each well, the system can be described by a single-band Bose-Hubbard model on a square lattice with a complex hopping matrix element:

$$
H_{\text {Hubbard }}=H^{(0)}+V
$$

with

$$
\begin{aligned}
H^{(0)} & =\frac{U}{2} \sum_{i} a_{i}^{\dagger} a_{i}\left(a_{i}^{\dagger} a_{i}-1\right)-\mu \sum_{i} a_{i}^{\dagger} a_{i} \\
V & =-t \sum_{\langle i j\rangle}\left(e^{i \phi_{i j}} a_{j}^{\dagger} a_{i}+e^{-i \phi_{i j}} a_{i}^{\dagger} a_{j}\right),
\end{aligned}
$$

where $a_{i}$ and $a_{i}^{\dagger}$ are bosonic field operators on site $i, U$ is the repulsive energy of two atoms in one well, $\mu$ is the chemical potential and $\langle i j\rangle$ denotes nearest-neighbor sites $i$ and $j$. The complex tunneling couplings appear in the Hubbard Hamiltonian due to the presence of the effective vector potential $\vec{A}$ that introduces an Aharonov-Bohm phase for the boson hopping from site to site. When an atom moves from a lattice site at $\vec{r}_{i}$ to a neighbouring site at $\vec{r}_{j}$, it will gain an Aharonov-Bohm phase:

$$
\phi_{i j}=\int_{\vec{r}_{i}}^{\vec{r}_{j}} \vec{A} \cdot d \vec{r}
$$

For a rotating lattice $\vec{A}=m \vec{\Omega} \times \vec{r} / \hbar$, where $\vec{\Omega}$ is the rotation frequency and $m$ is the mass of the atom. We consider the case of the uniform magnetic field $\vec{B}=\vec{\nabla} \times \vec{A}=$ $B \hat{z}$. The vector potential introduces frustration in the atomic motion if the phase twists around each plaquette add $\mathrm{p}$ to $2 \pi \alpha$ for some noninteger $\alpha$. The frustration parameter $\alpha$ is defined as the flux per plaquette in units of $2 \pi$ :

$$
\alpha=\frac{1}{2 \pi} \int \vec{B} \cdot d \vec{S}_{\mathrm{plaq}}=\frac{1}{2 \pi} \sum_{\mathrm{plaq}} \phi_{i j}
$$

where the integration is over the surface of a lattice plaquette and the sum is performed anticlockwise over the edges of the square plaquette. Due to the periodicity un$\operatorname{der} \alpha \rightarrow \alpha+1$ we can restrict the values of $\alpha$ to $0 \leq \alpha<1$. 
The frustration is maximal at $\alpha=1 / 2$. We choose the Landau gauge $\vec{A}=B(0, x, 0)$ so that the AharonovBohm phase $\phi_{i j}$ is zero on all horizontal bonds of the lattice, $\phi_{(x, y)(x \pm 1, y)}=0$, and $\phi_{(x, y)(x, y \pm 1)}= \pm 2 \pi \alpha x$.

We further focus on the limit of weak tunneling compared to the repulsive energy $U$ for two atoms in one well, $t \ll U$, where strong interparticle repulsion can lead to strongly correlated ground states. In such a limit the site occupation can be restricted to zero and one boson and the Bose-Hubbard Hamiltonian (11) can be mapped onto a spin- $1 / 2$ frustrated $X Y$ model. The two $S_{z}$ states of the pseudospin at a lattice site $i$ correspond to whether a lattice contains a boson or not. The strongly correlated phases of the system that we study in the present paper will correspond to spin-liquid phases of the effective frustrated quantum spin model.

The mapping of the Hubbard Hamiltonian (1) in the limit of $t \ll U$ to an effective spin Hamiltonian is possible because hard-core boson operators have the same commutation relations as spin- $1 / 2$ operators. The operators on different sites commute and operators on the same site anticommute. The spin raising and lowering operators correspond to the creation and annihilation operators of hard-core bosons and the motion of the atoms translates to pseudospin exchange. The effective spin Hamiltonian is:

$$
H_{e f f}=-\frac{J}{2} \sum_{\langle i j\rangle}\left(e^{i \phi_{i j}} S_{i}^{+} S_{j}^{-}+e^{-i \phi_{i j}} S_{j}^{+} S_{i}^{-}\right)-h \sum_{i} \hat{S}_{i}^{z}
$$

where $J=2 t, S_{i}^{ \pm}=S_{i}^{x} \pm i S_{i}^{y}$ are spin- $1 / 2$ operators, and $h=\mu$ represents an effective Zeeman field. Note that this is a ferromagnet in the absence of frustration $\left(\phi_{i j}=0\right)$. Also, it can be easily verified that the Hamiltonian (5) has local gauge invariance. If we change the gauge, $\vec{A} \rightarrow \vec{A}+\vec{\nabla} \chi$, then the Hamiltonian stays unchanged if the boson and spin operators pick up a phase change, $\phi_{i j} \rightarrow e^{i\left(\chi_{j}-\chi_{i}\right)} \phi_{i j}, a_{i} \rightarrow e^{i \chi_{i}} a_{i}$ and $S_{i}^{-} \rightarrow e^{i \chi_{i}} S_{i}^{-}$. The change of gauge corresponds to a spin rotation of $\chi_{i}$ in the $x y$ plane.

In the following sections we study spin liquid phases of the frustrated quantum spin model (5) using Schwinger boson mean field theory and PSG analysis. As demonstrated in Sec. VI those spin liquid phases correspond to FQH states of the system.

\section{SCHWINGER BOSON MEAN FIELD THEORY}

Schwinger boson mean field theory (SBMFT) was first proposed by Arovas and Auerbach $\stackrel{40}{\underline{4}}$ for SU(2) invariant spin models and was further applied to frustrated antiferromagnets 41 and generalized to anisotropic spin models $\underline{\underline{42}}$ Within the SBMFT spin $-1 / 2$ operators are represented in terms of the Schwinger boson operators $a_{i \uparrow}$ and $a_{i \downarrow}$ as follows:

$$
\begin{aligned}
S_{i}^{z} & =\frac{1}{2}\left(a_{i \uparrow}^{\dagger} a_{i \uparrow}-a_{i \downarrow}^{\dagger} a_{i \downarrow}\right), \\
S_{i}^{+} & =a_{i \uparrow}^{\dagger} a_{i \downarrow}, S_{i}^{-}=a_{i \downarrow}^{\dagger} a_{i \uparrow} .
\end{aligned}
$$

In addition, the Schwinger bosons satisfy the local constraint

$$
a_{i \uparrow}^{\dagger} a_{i \uparrow}+a_{i \downarrow}^{\dagger} a_{i \downarrow}=1
$$

at every lattice site.

To derive a mean field theory that can describe both ordered and disordered phases, i.e. a mean field Hamiltonian that does not have any preferred direction in spin space, the first step is to rewrite a spin Hamiltonian in terms of bond operators which are bilinear forms in Schwinger boson operators from nearest-neighbor sites. Introduction of one complex Hubbard-Stratonovich field at each bond then provides a natural mean field decoupling scheme. There are usually many possible bond operators that one can define. However, the only relevant ones are the bond operators that are invariant under the transformations of the symmetry group of the Hamiltonian. The spin Hamiltonian (5) is invariant under spin rotations around the $z$-direction and has $U(1)$ symmetry. In that case one can define four different bond operators that are invariant under symmetry operations of the Hamiltonian:

$$
\begin{aligned}
F_{i j} & =e^{i \phi_{i j} / 2} a_{i \uparrow}^{\dagger} a_{j \uparrow}+e^{-i \phi_{i j} / 2} a_{i \downarrow}^{\dagger} a_{j \downarrow}, \\
A_{i j} & =e^{-i \phi_{i j} / 2} a_{i \uparrow} a_{j \downarrow}-e^{i \phi_{i j} / 2} a_{i \downarrow} a_{j \uparrow}, \\
X_{i j} & =e^{-i \phi_{i j} / 2} a_{i \uparrow} a_{j \downarrow}+e^{i \phi_{i j} / 2} a_{i \downarrow} a_{j \uparrow}, \\
Z_{i j} & =e^{i \phi_{i j} / 2} a_{i \uparrow}^{\dagger} a_{j \uparrow}-e^{-i \phi_{i j} / 2} a_{i \downarrow}^{\dagger} a_{j \downarrow} .
\end{aligned}
$$

It can be easily verified that defined bond operators commute with the operator for the total magnetization in the $z$-direction, $\hat{M}=\sum_{i} S_{i}^{z}$, and are thus $U(1)$ symmetric. These bond operators are analogous to the bond operators defined for the quantum $X X Z$ model ${ }^{42}$ with additional phase factors which reflect the presence of an effective vector potential.

Nonzero expectation values for these bond operators signal the presence of various short-range correlations, that is, indicate the presence of short-range order. $F_{i j}$ and $A_{i j}$ are analogous to $S U(2)$ ferromagnetic and antiferromagnetic bond operators, respectively. The bond operators $X_{i j}$ and $Z_{i j}$ represent $X Y$ and easy axis correlations, respectively. Depending on the expected dominant correlations in the ground state of a given Hamiltonian, the Hamiltonian is further rewritten in terms of the products of appropriate bond operators.

For the Hamiltonian (5) at $h<h_{C}(\alpha)$, where $h_{C}(\alpha)$ is a critical value of the effective Zeeman field above which the system is in the Mott insulating phase, the classical ground state has a finite total magnetization in the $X Y$ plane $\frac{49}{}$ and the operators appropriate to describe the correlations in the ground state are $F_{i j}$ and $X_{i j}$. The 
Hamiltonian (5) can then be rewritten in the following form:

$$
\begin{aligned}
H & =\frac{J}{4} \sum_{\langle i j\rangle} n_{i} \cdot n_{j}+\frac{J z}{8} \sum_{i} n_{i} \\
& -\frac{J}{4} \sum_{\langle i j\rangle}\left(F_{i j}^{\dagger} F_{i j}+X_{i j}^{\dagger} X_{i j}\right)-h \sum_{i} S_{i}^{z},
\end{aligned}
$$

where $n_{i}=n_{i \uparrow}+n_{i \downarrow}=2 S$ is the number of bosons at each lattice site and is constant, and $z=4$ is the number of nearest neighbors. This representation can then be generalized to $N$ Schwinger boson flavors 40.43 and the functional integral representation of the partition function can be systematically expanded in powers of $1 / N$. The mean field Hamiltonian corresponds to a saddle point solution of the large $N$ action after Hubbard Stratonovich transformation. Since here we are interested only in the mean field solution, we do not use the large $N$ language. We assume that the bond operators have nonzero expectation values:

$$
\begin{aligned}
\left\langle F_{i j}\right\rangle & =f_{i j}, \\
\left\langle X_{i j}\right\rangle & =x_{i j},
\end{aligned}
$$

and perform a Hartree-Fock decomposition of the Hamiltonian (5):

$$
O_{i j}^{\dagger} O_{i j} \rightarrow o_{i j} O_{i j}^{\dagger}+o_{i j}^{*} O_{i j}-\left|o_{i j}\right|^{2},
$$

where $O_{i j} \in\left\{F_{i j}, X_{i j}\right\}$. The mean field Hamiltonian is then:

$$
\begin{aligned}
H_{M F} & =\frac{J}{4} \sum_{\langle i j\rangle}\left(\left|f_{i j}\right|^{2}+\left|x_{i j}\right|^{2}\right)+\frac{J}{4} \sum_{\langle i j\rangle} n_{i} \cdot n_{j}+\frac{J z}{8} \sum_{i} n_{i} \\
& -\frac{J}{4} \sum_{\langle i j\rangle}\left(f_{i j} \cdot F_{i j}^{\dagger}+f_{i j}^{*} \cdot F_{i j}+x_{i j} \cdot X_{i j}^{\dagger}+x_{i j}^{*} \cdot X_{i j}\right) \\
& -\frac{h}{2} \sum_{i}\left(n_{i \uparrow}-n_{i \downarrow}\right)+\sum_{i} \lambda_{i}\left(n_{i \uparrow}+n_{i \downarrow}-\kappa\right) .
\end{aligned}
$$

Within the mean field theory the constraint on the Schwinger boson occupation number at site $i$ is imposed on average:

$$
\left\langle n_{i \uparrow}+n_{i \downarrow}\right\rangle=2 S=\kappa,
$$

by introducing a chemical potential $\lambda_{i}$ and the average boson number $\kappa$ can also be taken as a parameter $\stackrel{43}{\underline{4}}$ Mean field theory parameters $f_{i j}$ and $x_{i j}$ are in general complex numbers called the mean field ansatz and can be found by solving self-consistent equations $\left\langle F_{i j}\right\rangle_{M F}=f_{i j}$ and $\left\langle X_{i j}\right\rangle_{M F}=x_{i j}$.

Within this method, magnetic ordering in the $X Y$ plane is identified as Bose condensation of the Schwinger bosons. In the presence of the magnetic ordering the energy excitation spectrum of Schwinger bosons is gapless, while a gapped excitation spectrum corresponds to incompressible spin liquid phases. Further we will focus on the possible noncondensed ground states of the system which are expected to be states without any spontaneously broken symmetry. As explained in one of the following sections, for such states we can use projective symmetry group analysis (PSG) to determine different mean field ansätze that reflect all the physical symmetries of the system, and to classify possible spin liquid states that can appear in the phase diagram of the system.

\section{MAGNETIC SYMMETRY GROUP}

Before proceeding to the PSG analysis we define a group of operators that correspond to the symmetries of the system and which commute with the Hamiltonian (5). The presence of an effective vector potential reduces the physical symmetries of the lattice and the Hamiltonian (5) does not commute with the standard lattice spatial symmetry operations. However, it is still possible to define operators that correspond to physical symmetries of the system. As will be described below, such operators obey the same multiplication relations as the original lattice spatial symmetry operators apart from additional phase factors and commute with the Hamiltonian (5). Generalized translation, rotation and reflection transformation operators in the presence of a uniform magnetic field can be specified and from such operators a full group can be constructed which is known as the magnetic symmetry group $\underline{\underline{47}, 50} \underline{\underline{52}}$

We define elementary translation $\left(T_{x}, T_{y}\right)$, rotation $(R)$ and reflection $(\tau)$ operators, illustrated in Fig. 1, in terms of their commutation relations with the spin operators $S_{i}^{ \pm}$. Since we have chosen the Landau gauge, the Hamiltonian (5) commutes with the translation operator in $y$ direction and the operator $T_{y}$ can be defined by

$$
T_{y} S_{(x, y)}^{+}=S_{(x, y+1)}^{+} T_{y} .
$$

It can be easily verified that $\left[H_{e f f}, T_{y}\right]_{-}=0$. However, the operator of a pure translation in $x$ direction does not commute with the Hamiltonian (5) due to the presence of $x$ dependent phase factors, and we therefore define a generalized unitary translation operator in $x$ direction as follows:

$$
T_{x} S_{(x, y)}^{+}=e^{-i 2 \pi \alpha y} S_{(x+1, y)}^{+} T_{x},
$$

where the phase factors in the previous equation are chosen to ensure $\left[H_{\text {eff }}, T_{x}\right]_{-}=0$. For $\alpha=p / q$ the Hamiltonian has periodicity $q$ in $x$ direction and $T_{x}^{q} S_{(x, y)}^{+}=$ $S_{(x+1, y)}^{+} T_{x}^{q}$. Similarly one can define a unitary rotation operator giving rotation by $\pi / 2$ counterclockwise around the origin:

$$
R S_{(x, y)}^{+}=e^{i 2 \pi \alpha x y} S_{(-y, x)}^{+} R,
$$

where the phase factor is again chosen to obtain $\left[H_{\text {eff }}, R\right]_{-}=0$. Also, an antiunitary operator $\tilde{\tau}$ that 


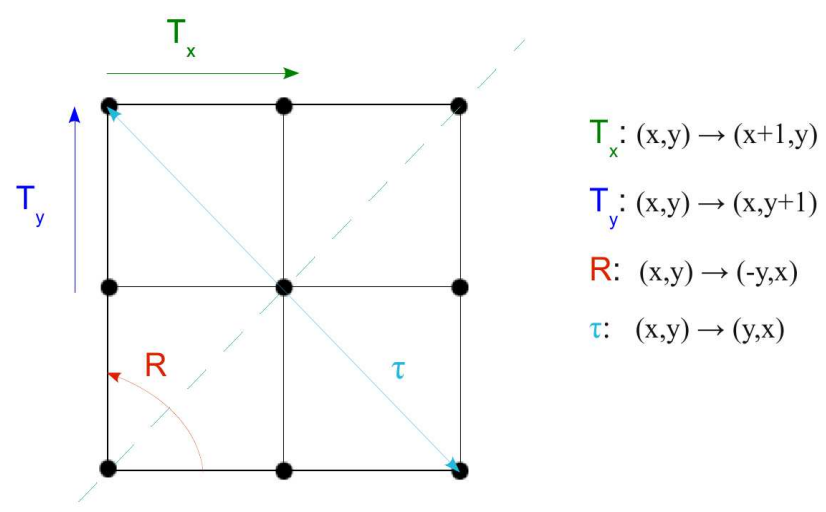

FIG. 1: (Color online) Symmetry operations (see text).

corresponds to reflection in the line $y=x$ can be defined,

$$
\tilde{\tau} S_{(x, y)}^{+}=e^{-i 2 \pi \alpha x y} S_{(y, x)}^{+} \tilde{\tau},
$$

which in combination with the antiunitary complex conjugation operator, $C$, forms a unitary operator and is a symmetry of the Hamiltonian (5):

$$
\tau \lambda S_{(x, y)}^{+}=\lambda^{*} e^{-i 2 \pi \alpha x y} S_{(y, x)}^{+} \tau,
$$

where $\tau=C \tilde{\tau}, \lambda$ is a complex number, and $\left[H_{e f f}, \tau\right]_{-}=$ 0 . The reflection operator itself is not a symmetry of the Hamiltonian since it reverses chirality while the magnetic field explicitly breaks chirality and time reversal symmetry. However, after combination with complex conjugation the appropriate sense of circulation is restored and operator $\tau$ denotes a symmetry of the Hamiltonian.

The magnetic symmetry group operators defined above have the same multiplication relations as ordinary spatial group operators apart from additional phase factors:

$$
\begin{aligned}
T_{x} T_{y} & =e^{i 2 \pi \alpha} T_{y} T_{x}, \\
T_{x} R & =R T_{y}^{-1}, R T_{x}=T_{y} R, R^{4}=1, \\
\tau T_{y} & =T_{x} \tau, \tau T_{x}=T_{y} \tau, \\
R \tau R \tau & =1, \tau \tau=1 .
\end{aligned}
$$

We also note that using the Schwinger boson representation of the spin operators (6), the magnetic symmetry group operators can also be defined in terms of their commutation relations with Schwinger bosons as follows:

$$
\begin{aligned}
T_{y} a_{(x, y) \sigma} & =a_{(x, y+1) \sigma} T_{y}, \\
T_{x} a_{(x, y) \sigma} & =e^{-i 2 \pi \alpha_{\sigma} y} a_{(x+1, y) \sigma} T_{x}, \\
R a_{(x, y) \sigma} & =e^{i 2 \pi \alpha_{\sigma} x y} a_{(-y, x) \sigma} R, \\
\tau \lambda a_{(x, y) \sigma} & =\lambda^{*} e^{-i 2 \pi \alpha_{\sigma} x y} a_{(y, x) \sigma} \tau,
\end{aligned}
$$

where $\lambda$ is a complex number, $\sigma \in\{\uparrow, \downarrow\}, \alpha_{\downarrow}=\alpha / 2, \alpha_{\uparrow}=$ $-\alpha / 2$ and $\alpha=\alpha_{\downarrow}-\alpha_{\uparrow}$, which is a definition convenient for PSG analysis. The equations (19) reflect the structure of the magnetic space group and will play a crucial role in determining possible mean field ansätze using PSG analysis as described in the following section.

\section{PROJECTIVE SYMMETRY GROUP ANALYSIS}

In this section we discuss possible spin liquid phases of the Hamiltonian (12). Spin liquid states are spin disordered states that typically appear in frustrated spin models $\stackrel{44.45}{.40}$ study and classify possible spin liquid phases we use a symmetry based analysis, PSG analysis, originally introduced by Wen and collaborators $\underline{46}-\underline{48}$ Within this theory we show that FQH states induced by a lattice potential, $\stackrel{13}{\rightleftharpoons}$ and with no counterpart in the continuum, correspond to " $\pi$ flux" spin liquid states of the effective frustrated spin model. To the best of our knowledge the connection between the spin liquid phases of the effective spin Hamiltonian and FQH states for bosonic atoms in rotating optical lattices has not been previously investigated.

As we are interested in the phases of the system that do not break any symmetries, we require that the Schwinger boson mean field theory reflects all the underlying microscopic symmetries of the spin model (5). This will lead to symmetric spin liquid states. The symmetry transformations include magnetic symmetry group transformations and $U(1)$ spin rotation symmetry. Using PSG analysis one can then construct all symmetry allowed mean field ansätze. It is important, however, to note that the microscopic symmetries of the spin model can be preserved in the mean field state in some indirect way. As first noted by Wen and collaborators $\underline{46}-\underline{48}$ in the context of fermionic mean field theory of spin liquids, the mean field theory ansatz should be invariant under transformations that are combined physical symmetry and gauge group transformations. This idea was then generalized to Schwinger boson mean field theory for spin liquid states by Wang and Vishwanath 44.45 The transformations that leave the mean field ansatz invariant then form a group called projective symmetry group (PSG). To find PSG transformations we first note that (spin independent) local $U(1)$ gauge transformations of Schwinger bosons

$$
a_{i \sigma} \rightarrow e^{i \phi_{i}} a_{i \sigma},
$$

leave the Hamiltonian (5) and all physical observables unchanged. However, the mean field ansatz is not invariant with respect to transformations (21) and transforms as follows:

$$
\begin{aligned}
F_{i j} & =e^{i\left(\phi_{j}-\phi_{i}\right)} F_{i j}, \\
X_{i j} & =e^{i\left(\phi_{i}+\phi_{j}\right)} X_{i j} .
\end{aligned}
$$

Since the physical spin state should be gauge invariant we see that that two different mean field ansätze that are related by a gauge transformation should, after implementing constraint (13) (that is, after projection to the 
physical spin space) lead to the same spin state. In other words, different mean field ansätze may correspond to the same physical state and the underlying symmetries may not be explicitly present in the mean field ansatz. Presence of the local $U(1)$ gauge transformations then explains why invariance of the mean field ansatz with respect to all microscopic symmetries is not a necessary condition to obtain symmetric spin liquid states. If the transformed (for example lattice translated) mean field ansatz is equivalent to $U(1)$ gauge transformed form of the ansatz the same spin state will be obtained after projection. Accordingly, invariance with respect to combined physical symmetry and gauge transformations is a sufficient condition to obtain symmetric states.

In addition to PSG transformations that are related to physical symmetries, one finds that there are also some elements of PSG that are pure local $U(1)$ gauge transformations. These transformations are not the result of a physical symmetry and can be associated with the emergent gauge group that describes obtained spin liquid phase. Such transformations form a subgroup of PSG which is called invariant gauge group (IGG). The microscopic theory of the spin liquid states of the system involves Schwinger boson spinons which are coupled to the emergent gauge field ${ }^{40,53,54}$ When the spinon spectrum is gapped and the emergent gauge field takes discrete values 54,55 ( $Z_{2}$ spin liquid), or emergent gauge theory is $U(1)$ gauge theory with a nontrivial topological term ${ }^{56}$, the mean field solution is stable and is a good starting point for identifying possible spin liquid states.

To examine the stability of a mean field solution one has to consider the fluctuations in $F_{i j}, X_{i j}$ and $\lambda_{i}$ that descibe the collective excitations above the mean field state. $F_{i j}$ and $X_{i j}$ have the amplitude and phase fluctuations. The amplitude fluctuations have a finite energy gap and are not important in discussing the stability and low energy properties of the possible spin liquid phases. The phase fluctuations of the mean field ansatz are gapped in the presence of a Chern-Simons term or spinon-pair condensation (Anderson-Higgs mechanism). Here the latter is achieved if both $F_{i j}$ and $X_{i j}$ are nonzero and in that case the emergent gauge theory is $Z_{2}$ (Ising) gauge theory. If $F_{i j}$ or $X_{i j}$ is zero the emergent gauge theory is $U(1)$ gauge theory. However, a Chern-Simons term present in the emergent gauge theory due to an effective uniform magnetic field gives the $U(1)$ gauge boson a nonzero gap. Gapped gauge bosons can mediate only short range interactions between spinons and stable spin liquid states always contain spinons with only short ranged interactions between spinons.

Further we will assume $Z_{2}$ spin liquid states, that is, we will initially assume that both $F_{i j}$ and $X_{i j}$ are nonzero. It is then clear that the only two elements of the IGG are the identity operation 1 and the IGG generator -1 . In other words, the only two transformations that leave the mean field ansatz invariant and are pure local $U(1)$ transformations (21) are $b_{i} \rightarrow e^{\phi_{i}} b_{i}$ with $\phi_{i}=0$ or $\phi_{i}=\pi$. The spin $U(1)$ rotation symmetry is already realized by con- sidering mean field ansatz, $X_{i j}$ and $F_{i j}$, of the form (8) which is explicitly invariant under spin rotation around $z$ direction. The operations that we further need to consider are magnetic symmetry group operations.

As discussed before we will consider symmetric spin liquid states that are invariant under PSG transformations. A PSG transformation will be a combined transformation of magnetic symmetry group and gauge transformations. However, it is not possible to combine a magnetic symmetry transformation with any gauge group transformation since algebraic relations (19) between magnetic symmetry group elements constrain the possible choices of gauge transformations. For each transformation $T\left(T \in\left\{T_{x}, T_{y}, R, \tau\right\}\right)$ in the magnetic symmetry group there is a gauge group transformation $G_{T}$ $\left(G_{T} \in\left\{G_{T_{x}}, G_{T_{y}}, G_{R}, G_{\tau}\right\}\right)$ such that the mean field ansatz is invariant under combined transformation $G_{T} T$. The gauge transformations can be represented as follows:

$$
G_{T}: b_{\vec{r} \sigma} \rightarrow e^{i \phi_{T}(\vec{r})} b_{\vec{r} \sigma} .
$$

The transformations $G_{T}$ can be found by considering algebraic constraints (19) (with $\alpha \rightarrow \alpha_{\sigma}$ when the magnetic symmetry group transformations are applied to the Schwinger boson operators $b_{i \sigma}$ ). For example, since $e^{i 2 \pi \alpha_{\sigma}} T_{x}^{-1} T_{y} T_{x} T_{y}^{-1}=1$, then $e^{i 2 \pi \alpha_{\sigma}}\left(G_{T_{x}} T_{x}\right)^{-1} G_{T_{y}} T_{y} G_{T_{x}} T_{x}\left(G_{T_{y}} T_{y}\right)^{-1}$ has to be equivalent to an identity operator, that is, it has to be an element of IGG (1 or -1$)$. Using relation

$$
Y^{-1} G_{X} Y b_{\vec{r} \sigma}\left(Y^{-1} G_{X} Y\right)^{-1}=e^{i \phi_{X}[Y(\vec{r})]} b_{\vec{r} \sigma},
$$

where here $X, Y \in\left\{T_{x}, T_{y}\right\}$, we obtain the following equation

$-\phi_{T_{x}}\left[T_{x}(\vec{r})\right]+\phi_{T_{y}}\left[T_{x}(\vec{r})\right]+\phi_{T_{x}}\left[T_{x} T_{y}^{-1}(\vec{r})\right]-\phi_{T_{y}}[\vec{r}]=p_{1} \pi$,

where $p_{1} \in\{0,1\}$ corresponds to IGG elements 1 and $-1\left(e^{i p_{1} \pi}\right.$ with $p_{1}=0$ or 1$)$.

Similar equations can be obtained by considering other algebraic relations in the equations (19). A detailed derivation of those equations is described in Appendix $\mathrm{A}$ and the most general solution that satisfies the obtained equations is:

$$
\begin{aligned}
\phi_{T_{x}}[x, y] & =0 \\
\phi_{T_{y}}[x, y] & =p_{1} \pi x \\
\phi_{\tau}[x, y] & =\phi_{\tau}[0,0]+p_{1} \pi x y, \\
\phi_{R}[x, y] & =\phi_{R}[0,0]+p_{1} \pi x y,
\end{aligned}
$$

where $p_{1} \in\{0,1\}$. If the nearest neighbour amplitudes $x_{i j}$ are nonzero then $\phi_{R}[0,0]=\phi_{\tau}[0,0]=p_{2} \pi / 2$ with $p_{2} \in\{0,1\}$. Possible mean field ansätze can then be obtained by requiring invariance of the mean field ansatz with respect to PSG transformations:

$$
\begin{aligned}
\left\langle G_{T} T F_{i j}\left(G_{T} T\right)^{-1}\right\rangle & =\left\langle F_{i j}\right\rangle=f_{i j}, \\
\left\langle G_{T} T X_{i j}\left(G_{T} T\right)^{-1}\right\rangle & =\left\langle X_{i j}\right\rangle=x_{i j},
\end{aligned}
$$


where $i$ and $j$ are nearest neighbours, $T \in\left\{T_{x}, T_{y}, R, \tau\right\}$ and $f_{i j}$ and $x_{i j}$ are both real and nonzero in general (Appendix A). From the conditions (27) we obtain four possible mean field ansätze:

$$
\begin{aligned}
A: f_{\left(x_{i}, y_{i}\right)\left(x_{i}+1, y_{i}\right)} & =f_{\left(x_{i}, y_{i}\right)\left(x_{i}, y_{i}+1\right)}=f, \\
x_{\left(x_{i}, y_{i}\right)\left(x_{i}+1, y_{i}\right)} & =x_{\left(x_{i}, y_{i}\right)\left(x_{i}, y_{i}+1\right)}=x, \\
B: f_{\left(x_{i}, y_{i}\right)\left(x_{i}+1, y_{i}\right)} & =f_{\left(x_{i}, y_{i}\right)\left(x_{i}, y_{i}+1\right)}=f, \\
x_{\left(x_{i}, y_{i}\right)\left(x_{i}+1, y_{i}\right)} & =-x_{\left(x_{i}, y_{i}\right)\left(x_{i}, y_{i}+1\right)}=x, \\
C: f_{\left(x_{i}, y_{i}\right)\left(x_{i}, y_{i}+1\right)} & =(-1)^{y_{i}} f_{\left(x_{i}, y_{i}\right)\left(x_{i}+1, y_{i}\right)}=f, \\
x_{\left(x_{i}, y_{i}\right)\left(x_{i}, y_{i}+1\right)} & =(-1)^{y_{i}} x_{\left(x_{i}, y_{i}\right)\left(x_{i}+1, y_{i}\right)}=x, \\
D: f_{\left(x_{i}, y_{i}\right)\left(x_{i}, y_{i}+1\right)} & =(-1)^{y_{i}} f_{\left(x_{i}, y_{i}\right)\left(x_{i}+1, y_{i}\right)}=f, \\
-x_{\left(x_{i}, y_{i}\right)\left(x_{i}, y_{i}+1\right)} & =(-1)^{y_{i}} x_{\left(x_{i}, y_{i}\right)\left(x_{i}+1, y_{i}\right)}=x,
\end{aligned}
$$

where $f_{i j}=f_{j i}^{*}, x_{i j}=x_{j i}, f_{i j}, x_{i j} \in \mathbb{R}$ and above equations are valid for all lattice sites $i=\left(x_{i}, y_{i}\right)$. The mean field ansätze above, illustrated in Fig. 2 then give local minima of the mean field Hamiltonian. The ansätze $A$ and $B$ can be distinguished from $C$ and $D$ by the emergent gauge invariant flux through an elementary square plaquette defined as the gauge invariant phase of the following products $\frac{57}{}$ :

$$
\begin{gathered}
f_{i j} f_{j k} f_{k l} f_{l i}=|f|^{4} e^{i \Phi}, \\
x_{i j} x_{j k} x_{k l} x_{l i}=|x|^{4} e^{i \Phi},
\end{gathered}
$$

with $i, j, k, l$ being sites of a square lattice plaquette. For the ansätze $A$ and $B$ the phase $\Phi=0$ (zero-flux states) and for $C$ and $D$ the phase $\Phi=\pi$ ( $\pi$-flux states) for all square lattice plaquettes. Therefore these two sets of states are clearly not gauge equivalent meanfield states. Also, $A$ and $B$ differ by the symmetry of the Schwinger boson pairing order parameter $x_{i j}$. Unlike for ansatz $A$, for ansatz $B$ the order parameter $x_{i j}$ changes sign under a $\pi / 2$ rotation ( $d$ wave like state). For $\pi$-flux states, $C$ and $D$, the unit cell for Schwinger bosons is doubled due to the emergent $\pi$ flux through each unit square plaquette of the lattice and the translation symmetry is not explicit in the mean field Hamiltonian. Similarly as for the ansätze $A$ and $B$, ansätze $C$ and $D$ differ by the symmetry of the order parameter $x_{i j}$. In the following section we further study possible noncondensed phases arising from the ansätze described previously and relate these states to bosonic fractional quantum Hall states for bosonic atoms in rotating optical lattices $\underline{13}$

\section{BOSONIC FRACTIONAL QUANTUM HALL STATES}

To further study noncondensed phases of the mean field Hamiltonian we take into account the local constraint (7) on Schwinger boson number at each site

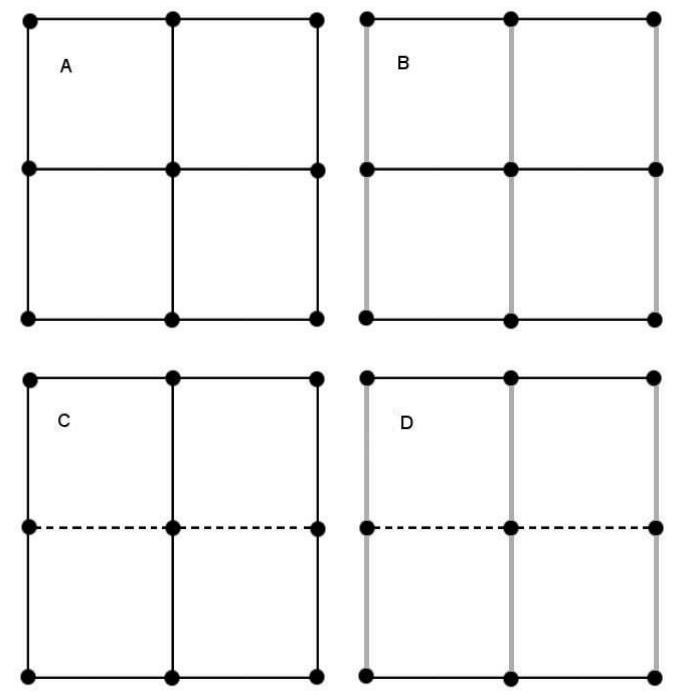

FIG. 2: The zero-flux (A and B) and the $\pi$-flux (C and D) mean field ansätze. The solid black, solid gray and dashed lines indicate bonds with $f_{i j}=f$ and $x_{i j}=x, f_{i j}=f$ and $x_{i j}=-x$ and $f_{i j}=-f$ and $x_{i j}=-x$, respectively. Here $f_{i j}=f_{j i}^{*}, x_{i j}=x_{j i}$ and both $f$ and $x$ are real.

exactly, $\stackrel{58-61}{\underline{6}}$ unlike in the conventional Schwinger boson mean field theory where the constraint is imposed on average as in the equation (13). The local constraint (7) introduces strong correlations between spin-up and spindown bosons. First we note that the constraint implies that the number of up/down bosons at each site can be only zero or one. Thus we require that the Schwinger boson operators satisfy the following anticommutation relations on the same site:

$$
\left\{a_{i, \sigma}, a_{i, \sigma^{\prime}}\right\}_{+}=0,\left\{a_{i, \sigma}, a_{i, \sigma^{\prime}}^{\dagger}\right\}_{+}=\delta_{\sigma, \sigma^{\prime}}
$$

where $\sigma, \sigma^{\prime} \in\{\uparrow, \downarrow\}$, and commutation relations

$$
\left[a_{i, \sigma}, a_{j, \sigma^{\prime}}\right]_{-}=0,\left[a_{i, \sigma}, a_{j, \sigma^{\prime}}^{\dagger}\right]_{-}=0,
$$

for the lattice sites $i$ and $j$ when $i \neq j$. The requirement above corresponds to a hard-core limit for each bosonic species and excludes states with more than one boson of the same species at the same lattice site. However, to take the constraint (7) into account exactly we also need to exclude the states with one spin-up and one spin-down Schwinger boson at the same lattice site. This is achieved by the Gutzwiller projection:

$$
P_{G}=\prod_{i}\left(1-n_{i \uparrow} n_{i \downarrow}\right),
$$

where $n_{i \uparrow}$ and $n_{i \downarrow}$ are the number operators at a site $i$ for spin-up and spin-down Schwinger bosons respectively, which for hard-core bosons have eigenvalues 0 or 1 . The ground state wave function is then

$$
\psi=P_{G} \psi_{M F},
$$


where $\psi_{M F}$ is the mean field Hamiltonian ground state wave function. To apply the Gutzwiller projection explic- itly it is convenient to rewrite the mean field Hamiltonian (12) in the following form:

$$
\begin{aligned}
H_{M F}= & h_{0}+\frac{h}{2} \sum_{i}\left(p_{i \downarrow}^{\dagger} p_{i \downarrow}+h_{i \uparrow}^{\dagger} h_{i \uparrow}\right)-\frac{J}{4} \sum_{\langle i j\rangle} f_{i j}\left\{e^{i \phi_{i j} / 2}\left(p_{i \downarrow} p_{j \downarrow}^{\dagger}+h_{i \uparrow} h_{j \uparrow}^{\dagger}\right)+e^{-\phi_{i j} / 2}\left(p_{j \downarrow} p_{i \downarrow}^{\dagger}+h_{j \uparrow} h_{i \uparrow}^{\dagger}\right)\right\} \\
& -\frac{J}{4} \sum_{\langle i j\rangle} x_{i j}\left\{e^{i \phi_{i j} / 2}\left(p_{i \downarrow} h_{j \uparrow}^{\dagger}+h_{i \uparrow} p_{j \downarrow}^{\dagger}\right)+e^{-i \phi_{i j} / 2}\left(h_{j \uparrow} p_{i \downarrow}^{\dagger}+p_{j \downarrow} h_{i \uparrow}^{\dagger}\right)\right\},
\end{aligned}
$$

where

$h_{0}=\frac{J}{4} \sum_{\langle i j\rangle}\left(\left|f_{i j}\right|^{2}+\left|x_{i j}\right|^{2}\right)+\frac{J}{4} \sum_{\langle i j\rangle} n_{i} \cdot n_{j}+\frac{J z}{8} \sum_{i} n_{i}-N_{s} \frac{h}{2}$,

with $N_{s}$ being the number of lattice sites, and where we have introduced the notation $p_{i \downarrow}=a_{i \downarrow}$ and $h_{i \uparrow}=a_{i \uparrow}^{\dagger}$. We have also taken into account that $f_{i j}$ and $x_{i j}$ are real for all mean field ansätze (28). The operator $h_{i \uparrow}^{\dagger}$ creates a spin-up bosonic hole and the operator $p_{i \downarrow}^{\dagger}$ creates a spindown bosonic particle at the site $i$. The constraint (7) can then be rewritten in the form:

$$
p_{i \downarrow}^{\dagger} p_{i \downarrow}=h_{i \uparrow}^{\dagger} h_{i \uparrow}
$$

where we have used the anticommutation relations (30). The local constraint (35) is equivalent to saying that wherever there is a spin-down boson there is also a spinup hole. The Gutzwiller projected wave function with $N_{\downarrow}$ spin-down bosons and $N-N_{\downarrow}$ spin-up bosons can then be obtained in the following way. Starting from the state with $N$ spin-up bosons, first $N_{\downarrow}$ spin-up bosons are annihilated and then $N_{\downarrow}$ spin-down bosons created at the coordinates of the spin-up holes. Therefore the wave function in the coordinate representation will be a function of the coordinates of spin-down bosons (or equivalently of spin-up bosonic holes) only.

Before examining further the effect of the Gutzwiller projection we note that the Hamiltonian (34) describes correlated hopping of particle-hole pairs. We are thus led to introduce the following hard core boson operators:

$$
b_{i \pm}=\frac{1}{\sqrt{2}}\left(p_{i \downarrow} \pm h_{i \uparrow}\right)
$$

It can be easily verified that these operators satisfy mixed commutation - anticommutation relations of the form (30) and (31). In terms of operators (36) the mean field
Hamiltonian (34) can be written as:

$$
\begin{gathered}
H_{M F}=h_{0}+\frac{h}{2} \sum_{i}\left(b_{i+}^{\dagger} b_{i+}+b_{i-}^{\dagger} b_{i-}\right) \\
-\frac{J}{4} \sum_{\langle i j\rangle} t_{i j}^{+}\left\{e^{i \phi_{i j} / 2} b_{i+} b_{j+}^{\dagger}+e^{-i \phi_{i j} / 2} b_{j+} b_{i+}^{\dagger}\right\} \\
-\frac{J}{4} \sum_{\langle i j\rangle} t_{i j}^{-}\left\{e^{i \phi_{i j} / 2} b_{i-} b_{j-}^{\dagger}+e^{-i \phi_{i j} / 2} b_{j-} b_{i-}^{\dagger}\right\},
\end{gathered}
$$

where $t_{i j}^{ \pm}=f_{i j} \pm x_{i j}$. Also, the constraint (35) implies the following condition:

$$
b_{i+}^{\dagger} b_{i+}=b_{i-}^{\dagger} b_{i-} .
$$

This can be seen from the equation $b_{i+}^{\dagger} b_{i+}-b_{i-}^{\dagger} b_{i-}=$ $p_{i \downarrow}^{\dagger} h_{i \uparrow}+h_{i \uparrow}^{\dagger} p_{i \downarrow}$. When the constraint (35) is taken into account explicitly then the only allowed states are the states with zero or one particle-hole pairs at a lattice site. Within this restricted single site basis terms $p_{i \downarrow}^{\dagger} h_{i \uparrow}$ and $h_{i \uparrow}^{\dagger} p_{i \downarrow}$ can be set to zero and condition (38) is obtained. The ground state wave function can then be obtained through the following projection:

$$
\psi\left(\left\{\vec{r}_{i}\right\}\right)=\left\langle 0\left|\prod_{i=1}^{N} h_{i \uparrow} p_{i \downarrow}\right| M F\right\rangle=\left\langle 0\left|\prod_{i=1}^{N} b_{i+} b_{i-}\right| M F\right\rangle,
$$

where $|0\rangle$ represents the vacuum of spin-down bosons and spin-up holes, (or equivalently vacuum of $b_{+}$and $b_{-}$bosons), $\vec{r}_{i}$ are coordinates of $N$ bosonic atoms in the lattice, and $|M F\rangle$ is a mean field state of bosons $b_{+}$and $b_{-}$.

The effective flux per plaquette of the lattice for $b_{+} / b_{-}$ bosons is $\alpha_{e f f}^{ \pm}=\alpha_{e f f} / 2$, with $\alpha_{e f f}$ being the effective flux for the atoms. For the mean field ansätze $A$ and $B$ the effective flux $\alpha_{\text {eff }}^{ \pm}=\alpha / 2$, and for the mean field anzätze $C$ and $D \alpha_{e f f}^{ \pm}=\alpha / 2+1 / 2$. Since the number of atoms is the same as the number of $b_{+}\left(b_{-}\right)$bosons and $\alpha_{e f f}^{ \pm}=\alpha_{e f f} / 2$ the wavefunction can be written as:

$$
\psi_{\nu}\left(\left\{\vec{r}_{i}\right\}\right)=\left[\psi_{\nu^{\prime}=2 \nu}^{\prime}\left(\left\{\vec{r}_{i}\right\}\right)\right]^{2},
$$

where $\psi_{\nu^{\prime}}^{\prime}\left(\left\{\vec{r}_{i}\right\}\right)$ is the wavefunction for $b_{+} / b_{-}$bosons at filling fractions $\nu^{\prime}=n_{+} / \alpha_{\text {eff }}^{+}=n_{-} / \alpha_{e f f}^{-}=2 \nu=$ 
$2 n / \alpha_{e f f}$, with $n_{+} / n_{-}$being the average density of $b_{+} / b_{-}$ bosons per lattice site. Thus at an atom filling fraction $\nu$, the average density of atoms per lattice site is $n=\alpha \nu^{\prime} / 2$ for the mean field ansätze $A$ and $B$, and $n=(1 / 2 \pm \alpha / 2) \nu^{\prime}$ for the mean field ansätze $C$ and $D$, where we have taken into account the symmetries under $\alpha_{e f f}^{ \pm} \rightarrow 1-\alpha_{e f f}^{ \pm}$and $n \rightarrow 1-n$ (particle-hole symmetry on the lattice).

We further argue that the FQH states of bosonic atoms in a rotating optical lattice correspond to the incompressible states for $b_{+}-b_{-}$pairs. Without the constraint, two species of hard-core bosons, $b_{+}$and $b_{-}$, are independent of each other and the ground state wavefunction of the mean-field Hamiltonian (37) can be written as a product of the ground state wavefunctions for $b_{+}$and $b_{-}$bosons. For example, at an effective filling fraction $\nu_{\text {eff }}^{\prime}=1$ the ground state for each bosonic species $\left(b_{+}\right.$and $\left.b_{-}\right)$is predicted to be an incompressible state that, in the continuum limit, can be described by the bosonic Pfaffian state ${ }^{\underline{7}}$ The projected wavefunction (40) then corresponds to the $\nu_{e f f}=1 / 2 \mathrm{FQH}$ states for $b_{+}-b_{-}$pairs (or equivalently for particle-hole pairs).

At $\nu_{\text {eff }}^{\prime}=1$, that is $\nu_{\text {eff }}=1 / 2$, possible incompressible ground states are at $n=\alpha / 2$ (mean field ansätze $A$ and $B$ ) and $n=1 / 2 \pm \alpha / 2$ (mean field ansätze $C$ and $D$ ). The states with $n=1 / 2 \pm \alpha / 2$ are lattice induced FQH states, with no counterpart in the continuum, and within presented mean field theory, correspond to " $\pi$ flux" spin liquid states of the effective spin model (mean field ansätze $C$ and $D$ ) for which the hard-core bosons $b_{+}$and $b_{-}$see an effective flux $\alpha_{\text {eff }}^{ \pm}=1 / 2+\alpha / 2$. Such incompressible quantum Hall states have previously been found using the composite fermion approach and exact diago-

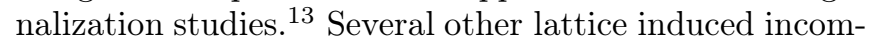
pressible states predicted within the composite fermion approach can also be related to incompressible states for $b_{+}-b_{-}$pairs, for example states at $\nu_{\text {eff }}^{\prime}=2 / 3$.

Finally, we note that the projected wavefunctions are also classified by PSG and that our results provide a guideline for a further search of possible ground states in the projected wavefunction space. We thus propose to study the effective spin model by Gutzwiller projected wavefunction variational approach ${ }^{62-64}$ as a possible direction of future research.

\section{CONCLUSIONS}

We have studied possible incompressible ground states for bosonic atoms in a rotating square optical lattice. In the hard-core boson limit the Bose-Hubbard Hamiltonian for the system can be mapped to a frustrated easy-plane magnet. Using Schwinger boson mean field theory and projective symmetry group analysis we have classified possible stable gapped spin liquid states of the effective spin model and related those states to FQH states for bosonic atoms in rotating optical lattices. In particular, we have found that, within the mean field theory devel- oped here, the FQH states induced by lattice potential and with no counterpart in the continuum limit, correspond to " $\pi$ flux" spin liquid states of the effective spin model. Since there are also competing condensed states on the lattice $\frac{12,49,65}{2}$ it is important to test the predictions of the mean field theory presented here. Finally, further work is necessary for more direct comparison of the obtained results with known results for FQH states in rotating optical lattices. We hope the Gutzwiller projected wavefunction variational approach will be further developed in future research.

\section{ACKNOWLEDGMENTS}

We thank Pedro Ribeiro for carefully reading the manuscript and for very helpful discussions. We also thank Paul McClarty and Dmitry Kovrizhin for very helpful discussions and suggestions.

\section{Appendix A: Solution to PSG equations}

In this appendix we derive equations for allowed gauge transformations $G_{T}$, defined by equation (23) in section V]

$$
G_{T}: b_{\vec{r} \sigma} \rightarrow e^{i \phi_{T}(\vec{r})} b_{\vec{r} \sigma} .
$$

As explained in section $\nabla$ the mean field ansatz should be invariant under combined transformations $G_{T} T$ where $T$ are magnetic symmetry group transformations. The transformations $G_{T}$ can be found by considering algebraic constraints (19) following the procedure explained in the example in the main text. We first describe in more details derivation of the equation (25) and then apply the same procedure to all other algebraic constraints given by equations (19).

From

$$
e^{i 2 \pi \alpha_{\sigma}} T_{x}^{-1} T_{y} T_{x} T_{y}^{-1}=1
$$

it follows

$$
e^{i 2 \pi \alpha_{\sigma}}\left(G_{T_{x}} T_{x}\right)^{-1} G_{T_{y}} T_{y} G_{T_{x}} T_{x}\left(G_{T_{y}} T_{y}\right)^{-1}=e^{i p_{1} \pi}
$$

where $p_{1} \in\{0,1\}$ corresponds to IGG elements 1 and -1 . The relation (A3) can be rewritten as

$$
\begin{aligned}
& T_{x}^{-1} G_{T_{x}}^{-1} T_{x} T_{x}^{-1} G_{T_{y}} T_{x} T_{x}^{-1} T_{y} T_{x} T_{y}^{-1} T_{y} T_{x}^{-1} G_{T_{x}} T_{x} T_{y}^{-1} G_{T_{y}}^{-1} \\
& =e^{i p_{1} \pi} e^{-i 2 \pi \alpha_{\sigma}} .
\end{aligned}
$$

Since $T_{x} T_{x}^{-1}=1$, and $T_{x}^{-1} T_{y} T_{x} T_{y}^{-1}=e^{-i 2 \pi \alpha_{\sigma}}$ and

$$
Y^{-1} G_{x} Y b_{\vec{r} \sigma}\left(Y^{-1} G_{x} Y\right)^{-1}=e^{i \phi_{X}[Y(\vec{r})]} b_{\vec{r} \sigma}
$$

we obtain equation (25):

$-\phi_{T_{x}}\left[T_{x}(\vec{r})\right]+\phi_{T_{y}}\left[T_{x}(\vec{r})\right]+\phi_{T_{x}}\left[T_{x} T_{y}^{-1}(\vec{r})\right]-\phi_{T_{y}}[\vec{r}]=p_{1} \pi$, 
with $p_{1} \in\{0,1\}$. We also note that, since $\phi_{T}$ are phases, all equations for phases in this section will be true modulo $2 \pi$. If a gauge transformation $b_{\vec{r} \sigma} \rightarrow e^{i \phi_{G}(\vec{r})} b_{\vec{r} \sigma}$ is applied then phases in (A1) change as follows:

$$
\phi_{T}(\vec{r}) \rightarrow \phi_{T}(\vec{r})+\phi_{G}(\vec{r})-\phi_{G}\left[T^{-1}(\vec{r})\right] .
$$

Using this gauge freedom the mean field ansatz can be made explicitly invariant under translation $T_{x}$ in $x$ direction, that is we can assume

$$
\phi_{T_{x}}(\vec{r})=0 .
$$

Then the equation (A6) becomes:

$$
\phi_{T_{y}}[x+1, y]-\phi_{y}[x, y]=p_{1} \pi .
$$

The solution of the equation above is then:

$$
\phi_{T_{y}}[x, y]=\phi_{T_{y}}[0, y]+p_{1} \pi x .
$$

We further assume that using gauge freedom we can set $\phi_{T_{y}}[0, y]=0$ while keeping $\phi_{T_{1}}(\vec{r})=0$. These two equations can be simultaneously satisfied for open boundary condition. We further assume open boundary condition in which case equation (A10) becomes:

$$
\phi_{T_{y}}[x, y]=p_{1} \pi x .
$$

Following the same procedure, from the algebraic constraints $T_{x}^{-1} R T_{y}^{-1} R^{-1}=1$ and $T_{y}^{-1} R T_{x} R^{-1}=1$ we obtain:

$$
\phi_{R}[x+1, y]-\phi_{R}[x, y]=p_{2}^{\prime} \pi+p_{1} \pi y,
$$

and

$$
\phi_{R}[x, y+1]-\phi_{R}[x, y]=p_{3}^{\prime} \pi+p_{1} \pi x,
$$

with $p_{2}^{\prime}, p_{3}^{\prime} \in\{0,1\}$. The solution of the above equations is:

$$
\phi_{R}[x, y]=\phi_{R}[0,0]+p_{2}^{\prime} \pi x+p_{3}^{\prime} \pi y+p_{1} \pi x y,
$$

Equivalently from $\tau T_{y}=T_{x} \tau$ and $\tau T_{x}=T_{y} \tau$ (or $\tilde{\tau} T_{y}=$ $T_{x} \tilde{\tau}$ and $\left.e^{i 4 \pi \alpha_{\sigma} y} \tilde{\tau} T_{x}=T_{y} \tilde{\tau}\right)$ we obtain following equations:

$$
\phi_{\tau}[x+1, y]-\phi_{\tau}[x, y]=p_{4}^{\prime} \pi+p_{1} \pi y
$$

and

$$
\phi_{\tau}[x, y+1]-\phi_{\tau}[x, y]=p_{5}^{\prime} \pi+p_{1} \pi x,
$$

with $p_{4}^{\prime}, p_{5}^{\prime} \in\{0,1\}$. The solution of the equations (A15) and (A16) is:

$$
\phi_{\tau}[x, y]=\phi_{\tau}[0,0]+p_{4}^{\prime} \pi x+p_{5}^{\prime} \pi y+p_{1} \pi x y .
$$

If we further apply the gauge transformation $\phi_{G}[x, y]=$ $\pi x$ the phases $\phi_{T}$ will change as follows:

$$
\begin{aligned}
\phi_{T_{x}}[x, y] & \rightarrow \phi_{T_{x}}[x, y]+\pi=\pi, \\
\phi_{T_{y}}[x, y] & \rightarrow \phi_{T_{y}}[x, y]=p_{1} \pi x, \\
\phi_{R}[x, y] & \rightarrow \phi_{R}[x, y]+\pi(x-y)=\phi_{R}[0,0]+\left(p_{2}^{\prime}+1\right) \pi x \\
& +\left(p_{3}^{\prime}-1\right) \pi y+p_{1} \pi x y, \\
\phi_{\tau}[x, y] & \rightarrow \phi_{\tau}[x, y]+\pi(x-y)=\phi_{R}[0,0]+\left(p_{4}^{\prime}+1\right) \pi x \\
& +\left(p_{5}^{\prime}-1\right) \pi y+p_{1} \pi x y .
\end{aligned}
$$

This gauge transformation does not change $\phi_{T_{y}}(\vec{r})$, nor does it really change $\phi_{T_{x}}(\vec{r})$ since a site-independent constant $\pi$ can be added to any $\phi_{T}(\vec{r})$ because of the IGG structure. However, when the transformation is applied $p_{2}^{\prime}, p_{4}^{\prime} \rightarrow p_{2}^{\prime}+1, p_{4}^{\prime}+1$, and $p_{3}^{\prime}, p_{5}^{\prime} \rightarrow p_{3}^{\prime}-1, p_{5}^{\prime}-1$. Since $p_{i}^{\prime}=0$ and $p_{i}^{\prime}=1$ are gauge equivalent $\forall i \in\{2,3,4,5\}$ we can always assume $p_{2}^{\prime}=p_{3}^{\prime}=p_{4}^{\prime}=p_{5}^{\prime}=0$.

Then we can write:

$$
\begin{aligned}
\phi_{T_{x}}[x, y] & =0 \\
\phi_{T_{y}}[x, y] & =p_{1} \pi x, \\
\phi_{R}[x, y] & =\phi_{R}[0,0]+p_{1} \pi x y, \\
\phi_{\tau}[x, y] & =\phi_{\tau}[0,0]+p_{1} \pi x y .
\end{aligned}
$$

The allowed mean field ansätze can then be obtained by requireing invariance of the mean field ansatz with respect to PSG transformations:

$$
\begin{aligned}
\left\langle G_{T} T F_{i j}\left(G_{T} T\right)^{-1}\right\rangle & =\left\langle F_{i j}\right\rangle=f_{i j}, \\
\left\langle G_{T} T X_{i j}\left(G_{T} T\right)^{-1}\right\rangle & =\left\langle X_{i j}\right\rangle=x_{i j},
\end{aligned}
$$

where $i$ and $j$ are nearest neighbours and $T \in\left\{T_{x}, T_{y}, R, \tau\right\}$.

Considering further following equations for the $(0,0) \rightarrow(-1,0)$ bond: $\quad f_{(0,0)(-1,0)}=$ $e^{-i\left(\phi_{R}[0,1]+\phi_{R}[1,0]-2 \phi_{R}[0,0]\right)} f_{(0,0)(1,0)}=f_{(0,0)(1,0)}$ and $f_{(-1,0)(0,0)}=f_{(0,0)(1,0)}=f_{(0,0)(-1,0)}^{*}$ we see that $f_{(0,0)(-1,0)}=f_{(0,0)(-1,0)}^{*} \equiv f$. In other words $f_{(0,0)(-1,0)}$ has to be real. Also, a global phase rotation, that is, a gauge transformation $\phi_{G}(\vec{r})=\phi$ does not change any PSG elements (equation (A7)). This can be used to fix one of the $x_{i j}$ presented to be real and positive, for example, $x_{(0,0)(0,1)}=x_{(0,0)(0,1)}^{*} \equiv x$.

If we assume that the nearest neighbour amplitudes $x_{i j}$ are nonzero then $\phi_{R}[0,0]$ can be found from the following equation for the $(0,0) \rightarrow(-1,0)$ bond: $x_{(0,0)(-1,0)}=e^{-i\left(2 \phi_{R}[0,0]+\phi_{R}[1,0]+\phi_{R}[0,1]\right)} x_{(0,0)(1,0)}$, which in combination with the equations $x_{(0,0)(-1,0)}=x_{(-1,0)(0,0)}=x_{(0,0)(1,0)}$ (due to the symmetry $x_{i j}=x_{j i}$ and translational invariance of $x_{i j}$ in $x$ direction) gives $4 \phi_{R}[0,0]=0$, that is, $\phi_{R}[0,0]=p_{2} \pi / 2$ with $p_{2} \in\{0,1\}$. Equivalently from $x_{(0,0)(0,1)}=e^{-i\left(\phi_{R}[0,0]+\phi_{R}[1,0]\right)} x_{(0,0)(1,0)}=$ $e^{-i\left(\phi_{\tau}[0,0]+\phi_{\tau}[-1,0]\right)} x_{(0,0)(-1,0)}$ we obtain $\phi_{\tau}[0,0]=$ $\phi_{R}[0,0]$. The algebraic constraints $R^{4}=1, R \tau R \tau=1$ and $\tau \tau=1$ do not further restrict possible values 
of $p_{1}$ and $p_{2}$. The mean field ansätze (27) can then be obtained from equations (A19) and A20 with
$\phi_{R}[0,0]=\phi_{\tau}[0,0]=p_{2} \pi / 2$.
1 M. Lewenstein, A. Sanpera, V. Ahufinger, B. Damski, A. Sen (De), and U. Sen, Adv. Phys. 56, 234 (2007)

2 D. Jaksch, and P. Zoller, New J. Phys. 5, 56 (2003)

3 M. Greiner, O. Mandel, T. Esslinger, T. W. Haensch, and I. Bloch, Nature (London) 415, 39 (2002)

${ }^{4}$ N. R. Cooper, Adv. Phys. 57, 539 (2008)

5 R. Bhat, M. Krämer, J. Cooper, and M. J. Holland, Phys. Rev. A 76, 043601 (2007)

6 A. S. Sørensen, E. Demler, and M. D. Lukin, Phys. Rev. Lett. 94, 086803 (2005)

7 N. K. Wilkin and J. M. F. Gunn, Phys. Rev. Lett. 84, 6 (2000)

8 N. R. Cooper, N. K. Wilkin, and J. M. F. Gunn, Phys. Rev. Lett. 87, 120405 (2001)

9 E. H. Rezayi, N. Read, and N. R. Cooper, Phys. Rev. Lett. 95, 160404 (2005)

10 R. N. Palmer, and D. Jaksch, Phys. Rev. Lett. 96, 180407 (2006)

11 M. Hafezi, A. S. Sørensen, E. Demler, and M. D. Lukin, Phys. Rev. A 76, 023613 (2007)

12 R. N. Palmer, A. Klein, and D, Jaksch, Phys. Rev. A 78, 013609 (2008)

13 G. Möler, and N. R. Cooper, Phys. Rev. Lett. 103,105303 (2009)

14 E. Kapit, and E. Mueller, Phys. Rev. Lett. 105, 215303 (2010)

15 R. O. Umucalilar, and E. J. Mueller, Phys. Rev. A 81, 053628 (2010)

16 K. v. Klitzing, G. Dorda, and M. Pepper, Phys. Rev. Lett. 45, 494 (1980)

17 D. C. Tsui, H. L. Stormer, and A. C. Gossard, Phys. Rev. Lett. 48, 1559 (1982)

18 D. C. Tsui, H. L. Störmer, and A. C. Gossard, Phys. Rev. B 25, 1405 (1982)

19 A. Y. Kitaev, Ann. Phys. (N.Y.) 303, 2 (2003)

20 S. Das Sarma, M. Freedman, C. Nayak, S. H. Simon, and A. Stern, Rev. Mod. Phys. 80, 1083 (2008)

21 B. Parades, P. Fedichev, J. I. Cirac, and P. Zoller, Phys. Rev. Lett. 87, 010402 (2001)

22 M. Polini, R. Fazio, A. M. MacDonald, and M. P. Tosi, Phys. Rev. Lett. 95, 010401 (2005)

23 S. Tung, V. Schweikhard, and E. A. Cornell, Phys. Rev. Lett. 97, 020402 (2006)

24 E. J. Mueller, Phys. Rev. A 70, 041603 (R) (2004)

25 C. Furtado, J. R. Nascimento, and L. R. Ribeiro, Phys. Lett. A 358, 336 (2006)

26 J. K. Pachos, Phys. Lett. A 344, 441 (2005)

27 J. K. Pachos and E. Rico, Phys. Rev. A 70, 053620 (2004)

28 A. Kay, D. K. K. Lee, J. K. Pachos, M. B. Plenio, M. E. Reuter, and E. Rico, Opt. Spectrosc. 99, 339 (2005)

29 G. Juzeliūnas, J. Ruseckas, P. Öhberg, and M. Fleischhauer, Phys. Rev. A 73, 025602 (2006)

${ }^{30}$ K. Osterloh, M. Baig, L. Santos, P. Zoller, and M. Lewenstein, Phys. Rev. Lett. 95, 010403 (2005)

31 J. Ruseckas, G. Juzeliūnas, P. Öhberg, and M. Fleis- chhauer, Phys. Rev. Lett. 95, 010404 (2005)

32 N. R. Cooper, Phys. Rev. Lett. 106, 175301 (2011)

33 N. R. Cooper, J. Dalibard, Europhys. Lett. 95, 66004 (2011)

34 J. E. Williams, and M. J. Holland, Nature (London) 401, 568 (1999)

35 D. Hofstadter, Phys. Rev. B 14, 2239 (1976)

36 R. O. Umucalilar, and M. Ö. Oktel, Phys. Rev. A 76, 055601 (2007)

37 R. B. Laughlin, Phys. Rev. Lett. 50, 1395 (1983)

38 N. Read, and E. Rezayi, Phys. Rev. B 59, 8084 (1999)

39 N. Regnault, C. C. Chang, Th. Jolicoeur, and J. K. Jain, J. Phys. B: At. Mol. Opt. Phys. 39, S89 (2006)

40 D. P. Arovas and A. Auerbach, Phys. Rev. B 38, 316 (1988)

41 P. Chandra, P. Coleman, and A. I. Larkin, J. Phys.: Condens. Matter 2, 7933 (1990)

42 A. A. Burkov and A. H. MacDonald, Phys. Rev. B 66, $115320(2002)$

43 S. Sachdev, Phys. Rev. B 45, 12377 (1992)

${ }^{44}$ F. Wang, and A. Vishwanath, Phys. Rev. B 74, 174423 (2006)

45 F. Wang, Phys. Rev. B 82, 024419 (2010)

46 X.-G. Wen, Phys. Lett. A 300, 175 (2002)

47 X.-G. Wen, Phys. Rev. B 65, 165113 (2002)

48 Y. Zhou, and X.-G. Wen, arXiv:0210662 (unpublished)

49 T. Đurić, and D. K. K. Lee, Phys. Rev. B 81, 014520 (2010)

50 E. Brown, Phys. Rev. 133 A1038 (1964)

51 J. Zak, Phys. Rev. 134, A10602 (1964); 134 A1607 (1964)

52 S. Powell, R. Barnett, R. Sensarma, and S. Das Sarma, Phys. Rev. A 83, 013612 (2011)

53 X. G. Wen, Phys. Rev. B 44, 2664 (1991)

54 N. Read, and S. Sachdev, Phys. Rev. Lett. 66, 1773 (1991)

55 R. Moessner, S. L. Sondhi, and E. Fradkin, Phys. Rev. B 65, 024504 (2001)

${ }^{56}$ M. A. Levin, and X. G. Wen, Phys. Rev. B 71, 045110 (2005)

57 O. Tchernyshyov, R. Moessner, and S. L. Sondhi, Europhys. Lett. 73, 278 (2006)

58 Z. Zou, B. Doucot, and B. S. Shastry, Phys. Rev. B 39, 11424 (1988)

59 K. Hallberg, A. G. Rojo, and C. A. Balseiro, Phys. Rev. B 42, 4827 (1990)

60 V. Ya. Krivnov, V. N. Likhachev, and A. A. Ovchinnikov, Phys. Lett. A 192, 425 (1994)

61 M.-C. Chang, and M.-F. Yang, Phys. Rev. B 66, 184416 (2002)

62 C. Gros, Ann. Phys. 189, 53 (1989)

63 Z.-X. Liu, Y. Zhou, H.-H. Tu, X.-G. Wen, and T.-K. Ng, Phys. Rev. B 85, 195144 (2012)

64 R. Sensarma, and V. Galitski, Phys. Rev. B 84, 060503(R) (2011)

${ }^{65}$ K. Kasamatsu, Phys. Rev. A 79, 021604(R) (2009) 